phys. stat. sol. (a) 184, No. 1, 105-110 (2001)

\title{
Structure and Energetics of P-rich GaP(001) Surfaces
}

\author{
O. Pulci ${ }^{1}$ ), W. G. Schmidt, and F. BeChSTEDT \\ Institut für Festkörpertheorie und Theoretische Optik, Friedrich-Schiller-Universität, \\ Max-Wien Platz 1, D-07743 Jena, Germany
}

(Received June 19, 2000; accepted January 2, 2001)

Subject classification: $68.35 . B s ; 68.35 . M d ;$ S7.11

Dedicated to Professor Dr. Wolfgang Richter on the occasion of his 60th birthday

The energetics and atomic structure of the P-rich $\mathrm{GaP}(001)$ surface are studied by first-principles total-energy calculations. For $(2 \times 2)$ reconstructed surfaces we predict the formation of a $\mathrm{P}$ bilayer, which is semiconducting due the formation of inequivalent dimers.

\section{Introduction}

The microscopic structure of the $\mathrm{GaP}(001)$ surface is not well understood, in marked contrast to other III-V compounds, in particular GaAs(001) (see Ref. [1] for a recent review). It has been suggested that ion bombardment and annealing of $\mathrm{GaP}(001)$ results in a $(4 \times 2)$ reconstructed Ga-rich surface $[2,3]$ in analogy to GaAs. On the other hand, based on ion scattering spectroscopy and low-energy electron diffraction (LEED) experiments, a $(2 \times 4)$ translational symmetry has been proposed [4, 5]. In solid-source molecular beam epitaxy a $(2 \times 4)$ diffraction pattern shows up under both $\mathrm{Ga}$ and $\mathrm{P}$ supply [6]. In addition, $(4 \times 4)$ spots have been observed.

A slightly different behaviour is found for samples grown by metal-organic vapour phase epitaxy or chemical beam epitaxy as well as for $\mathrm{GaP}(001)$ surfaces prepared by thermal desorption of a protective arsenic/phosphorous double layer cap under ultrahigh vacuum conditions [7-9]. After annealing to $690 \mathrm{~K}$ the de-capped $\mathrm{GaP}(001)$ surfaces show a $(2 \times 1) /(2 \times 2)$-like LEED pattern. Annealing to higher temperatures $(785 \mathrm{~K})$ leads to a $(2 \times 4)$ translational symmetry. The many-step annealing procedure is accompanied by a transition from a more P-rich to a more Ga-rich $\mathrm{GaP}(001)$ surface. Previous total-energy (TE) calculations $[8,9]$ show the stability of several $(2 \times 4)$ reconstructions. For Ga-rich surfaces they are characterized by single $\mathrm{Ga}-\mathrm{Ga}$ or mixed $\mathrm{Ga}-\mathrm{P}$ dimers on top of a complete Ga layer. For lower Ga contents one Ga pair is missing in this layer and one ( $\delta$ structure) or two P dimers ( $\beta 2$ structure) terminate the surface. $(4 \times 2)$ reconstructions with $\mathrm{Ga}$ or $\mathrm{P}$ dimers in the uppermost atomic layer are unstable. The comparison of measured and calculated reflectance anisotropy spectra support the mixed-dimer reconstruction of the Ga-rich $\mathrm{GaP}(001)$ surface [8, 9]. This has recently been confirmed by a comparison of calculated and measured scanning-tunneling microscopy $(\mathrm{STM})$ images [10]. The $(2 \times 4)$ mixed-dimer structure agrees with recent measurements of the surface core-level shifts [5].

The structure of the $(2 \times 1) /(2 \times 2)$ reconstruction of the $\operatorname{GaP}(001)$ surface remains unknown. Auger electron spectroscopy indicates a P-rich surface. The similarities with

1) e-mail: pulci@ifto.physik.uni-jena.de 
diffraction pattern of P-rich $\operatorname{InP}(001)$ surfaces $[9,11,12]$ indicate a surface terminated with $\mathrm{P}$ dimers. In this paper, the structure and the stability of $\operatorname{GaP}(001)(2 \times 2)$ is studied by means of TE calculations and compared to results for $(2 \times 4)$ reconstructed surfaces.

\section{Computational Methods}

The calculations are based on the density-functional theory in the local-density approximation (DFT-LDA). The electron-ion interaction is treated by using fully separable, norm-conserving pseudopotentials [13]. The $\mathrm{Ga} 3 \mathrm{~d}$ electrons are taken into account using a nonlinear core correction to the exchange and correlation energy. The electronic wave functions are expanded into plane waves up to an energy cutoff of $18 \mathrm{Ry}$. k-space integrations are performed using eight special points in the irreducible part of the Brillouin zone of the $(2 \times 2)$ reconstructions and equivalent sets of points for larger surface unit cells. The surfaces are modeled using periodic super-cells. Each cell includes an atomic slab with six to seven atomic layers and a vacuum region corresponding to five layers. The cation-terminated bottom layer of the slab is saturated with fractionally charged $\mathrm{H}$ atoms [14]. The two bottom layers are kept frozen, whereas all other atoms are allowed to relax. The minima of the TE functional with respect to both the atomic and electronic degrees of freedom are found by means of a molecular-dynamical approach [15]. The calculations are performed with the theoretical equilibrium lattice constant of $5.42 \AA$, slightly smaller than the measured value of $5.45 \AA$.

\section{Results and Discussion}

We studied the structural models shown in Fig. 1. These reconstructions realize P coverages between $\Theta=0$ and 2, where $\Theta=1$ corresponds to one complete monolayer (ML) of phosphorus on top of the Ga-terminated substrate. We consider the adsorption on this Ga layer in one $(i=1)$ or two $(i=2)$ adlayers. The reconstruction elements are $\mathrm{Ga}-\mathrm{P}$ mixed dimers (md) and buckled or unbuckled $\mathrm{P}-\mathrm{P}$ top dimers (td). In detail, we study a $(2 \times 1)$ md1 structure $(\Theta=0)$, with one mixed dimer per $(2 \times 1)$ cell in the first adlayer, a $(2 \times 1) \operatorname{td} 1$ one $(\Theta=1)$ with one P-dimer per $(2 \times 1)$ cell, a $(2 \times 2)$ td1 overlayer $\left(\Theta=\frac{1}{2}\right)$ with one top dimer in the first adlayer, and two different reconstructions $\mathrm{p}(2 \times 2) \mathrm{td} 1$ and $\mathrm{p}(2 \times 2) \mathrm{td} 1 \mathrm{~s}$ (where "s" indicates a staggered arrangement of dimers) with two top dimers in the first adlayer $(\Theta=1)$. For the more P-rich phase, we have studied one $(2 \times 2) \mathrm{td} 2$ geometry with one top dimer on a complete $\mathrm{P}$ adlayer $(\Theta=1.5)$, and two geometries $\mathrm{p}(2 \times 2) \mathrm{td} 2$ and $\mathrm{p}(2 \times 2) \mathrm{td} 2 \mathrm{~s}$ with two phosphorus adlayers $(\Theta=2)$. These surface models are compared with two reconstructions, the $(2 \times 4) \beta 2\left(\Theta=\frac{2}{3}\right)$, and the $(2 \times 4)$ mixed-dimer $(\Theta=0)$ which have been studied previously $[8,9,16]$.

The stability of the investigated structures is shown in a zero-temperature phase diagram (Fig. 2), where the surface energies are plotted versus the chemical potential of $\mathrm{Ga}$. The upper limit of $\mu(\mathrm{Ga})$ is given by its bulk value and has been calculated for $\mathrm{Ga}$ metal with orthorhombic structure. $\Delta H_{\mathrm{f}}(\mathrm{GaP})$ gives the thermodynamically allowed interval for the variation $\Delta \mu(\mathrm{Ga})$ (for details see, e.g., Ref. [17]). We use the experimental value of $0.91 \mathrm{eV}$ for $\Delta H_{\mathrm{f}}(\mathrm{GaP})$, which refers to white phosphorus [18]. We stress that the limit for the P-rich phase contains an uncertainty due to the fact that under the 


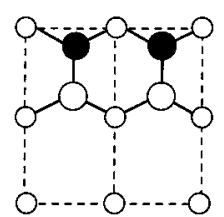

$(2 \times 1) \mathrm{md} 1$
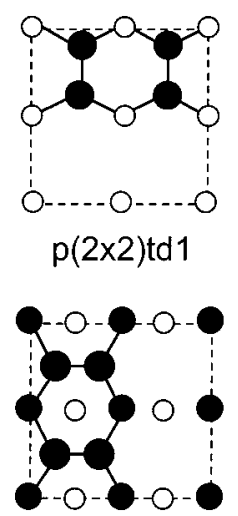

$p(2 \times 2) t d 2$

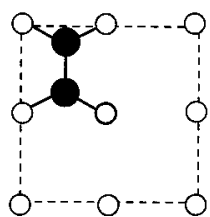

$(2 \times 2) \operatorname{td} 1$

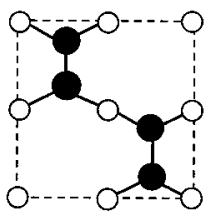

$p(2 \times 2) t d 1 s$

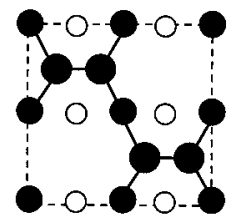

$p(2 \times 2) t d 2 s$

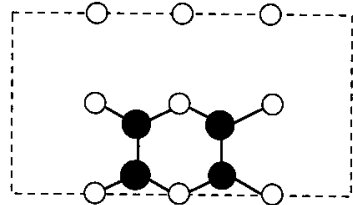

(2x4)ß2

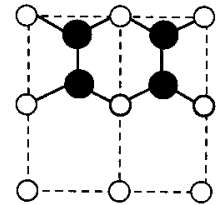

(2x1)td 1

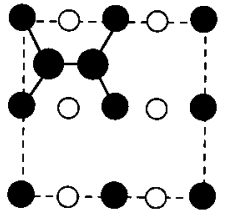

$(2 \times 2) \mathrm{td} 2$
[110]

Fig. 1. Top view of the geometries considered. Full circles: $\mathrm{P}$ atoms; open circles: Ga atoms

experimental growth conditions amorphous $\mathrm{P}$ and not white $\mathrm{P}$ is deposited on the top of the Ga surface, if no Ga atoms are supplied. Therefore, and in order to allow for numerical inaccuracies in the chemical potentials and to accommodate possible changes due to temperature effects, the interval of these variations is slightly extended.

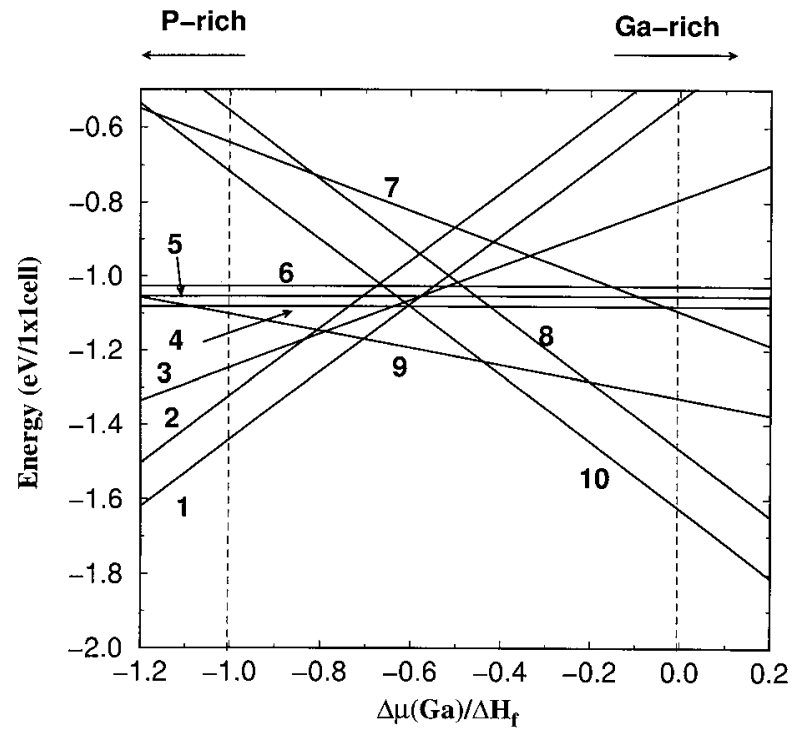

Fig. 2. Relative surface energy per $(1 \times 1)$ unit cell vs. the variation of the Ga chemical potential. (1) $\mathrm{p}(2 \times 2) \mathrm{td} 2$, (2) $\mathrm{p}(2 \times 2) \mathrm{td} 2 \mathrm{~s}$, (3) $(2 \times 2) \mathrm{td} 2, \quad(4) \mathrm{p}(2 \times 2) \mathrm{td} 1$, (5) $(2 \times 1) \mathrm{td} 1,(6) \mathrm{p}(2 \times 2) \mathrm{td} 1 \mathrm{~s}$, (7) $(2 \times 2) \mathrm{td} 1, \quad(8)(2 \times 1) \mathrm{md} 1$, (9) $(2 \times 4) \beta 2,(10)(2 \times 4)$ mixeddimer 
The phase diagram confirms earlier findings $[8,16]$ concerning the stabilization of mixed dimers on top of the Ga termination for more Ga-rich surface preparation conditions (line 10 in Fig. 2). The same holds for intermediate chemical potentials where the $(2 \times 4) \beta 2$ reconstruction is stable (line 9 in Fig. 2). For $\Delta \mu(\mathrm{Ga}) \approx 0.7 \Delta H_{\mathrm{f}}(\mathrm{GaP})$ a variety of surface structures are nearly degenerate in energy. In addition to the two $(2 \times 4)$ structures mentioned above the P-rich $\mathrm{p}(2 \times 2) \operatorname{td} 2$ structure $(\Theta=2)$ but also $\mathrm{P}$ monolayer coverages with different dimer arrangements $(\Theta=1)$ may occur. This indicates a strong tendency for disorder for that particular choice of the Ga chemical potential.

The calculated phase diagram is similar to the one of $\operatorname{InP}(001)$ [19], where also the $(2 \times 4)$ mixed dimer, the $(2 \times 4) \beta 2$ and the $\mathrm{p}(2 \times 2) \operatorname{td} 2$ are energetically favourable going from the cation-rich to the anion-rich phases.

Under the extreme P-rich conditions the $\mathrm{p}(2 \times 2) \mathrm{td} 2$ structure is favourable in agreement with the experimentally observed translational symmetries [8,9]. This structure represents two complete monolayers of phosphorus. The atoms in the uppermost atomic layer are dimerized. The dimers are arranged in linear chains parallel to the [110] direction. Compared to the zig-zag chain arrangement in the $\mathrm{p}(2 \times 2) \mathrm{td} 2 \mathrm{~s}$ structure (cf. Fig. 1) an energy of about $0.1 \mathrm{eV}$ per surface atom is gained. Our calculations show no evidence for dimer buckling. Rather, the semiconducting character of the $\mathrm{p}(2 \times 2) \mathrm{td} 2$ surface is a consequence of the structural inequivalence of the two topmost dimers as shown in Fig. 3. The dimers in the uppermost $\mathrm{P}$ layer are symmetric with respect to the

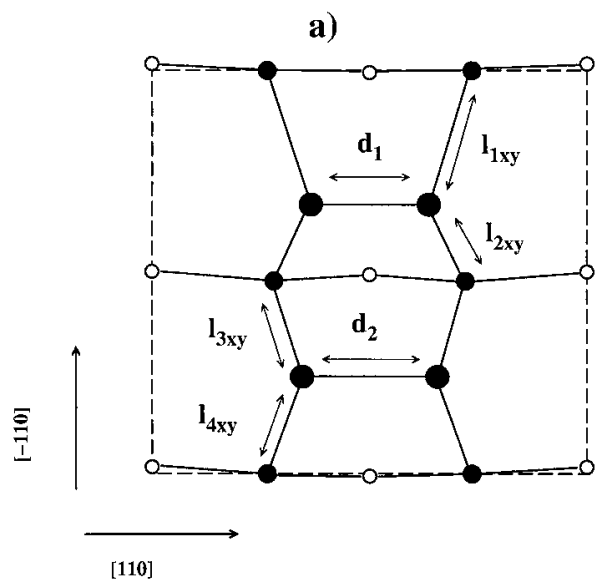

b)

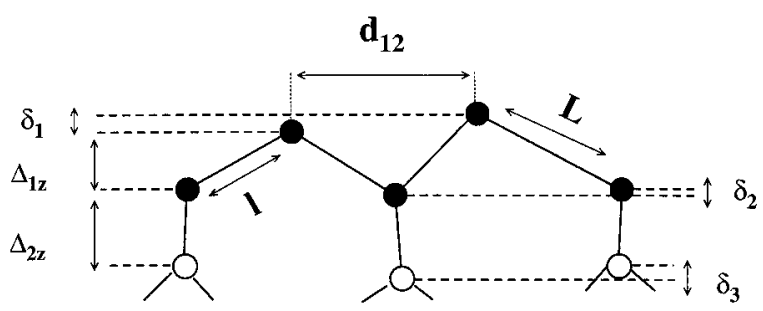

Fig. 3. a) Top and b) side view of the P-rich $\mathrm{GaP}(001) \mathrm{p}(2 \times 2) \mathrm{td} 2$ geometry. Open (full) circles represent Ga (P). Large circles indicate positions in the topmost layer. The bond lengths are given in Table 1 
Table 1

Structural parameters (in $\AA$ ) for $\mathrm{p}(2 \times 2) \mathrm{td} 2 \mathrm{GaP}(001)$ surface The parameters are explained in Fig. 3

\begin{tabular}{llllllllllllll}
\hline$d_{1}$ & $d_{2}$ & $l_{1 x y}$ & $l_{2 x y}$ & $l_{3 x y}$ & $l_{4 x y}$ & $\delta_{1}$ & $\delta_{2}$ & $\delta_{3}$ & $\Delta_{1 z}$ & $\Delta_{2 z}$ & $d_{12}$ & $L$ & $l$ \\
\hline 2.08 & 2.38 & 2.65 & 1.59 & 1.89 & 1.93 & 0.34 & 0.11 & 0.24 & 1.09 & 1.46 & 3.28 & 3.01 & 2.22 \\
\hline
\end{tabular}

[110] direction. The two P-P dimers occur at different vertical distances $\left(\Delta_{1 z}+\Delta_{2 z}\right)=$ $2.55 \AA$ and $\left(\Delta_{1 z}+\Delta_{2 z}+\delta_{1}\right)=2.89 \AA$ from the Ga-terminated bulk. They differ in their heights by $0.34 \AA$ (cf. Fig. 3). Also the dimer bonding is quite different. The upper dimer is characterized by a strong bond with a bond length $d_{1}=2.08 \AA$, whereas the lower one possesses a much weaker bonding with a bond length $d_{2}=2.38 \AA$. It is larger than the sum of the covalent radii of $2.12 \AA$ but still remarkably smaller than the lateral distance of $3.83 \AA$ of unbonded atoms in an ideal $\mathrm{GaP}(001)$ plane. The uppermost $\mathrm{P}$ dimer is considerably displaced parallel to [110] as indicated by the projection of the corresponding bond length $l_{1 x y}=2.65 \AA$. One of the back bonds is weakened as indicated by their different lengths $L=3.01 \AA$ and $l=2.22 \AA$. This can be interpreted as a tendency of $\mathrm{P}$ atoms in the layer beneath towards threefold coordination. Hence, the adlayer atoms show features known from solid black phosphorus, where the $\mathrm{P}$ atom has only three nearest neighbours at a distance of about $2.20 \AA$.

The structural rearrangements are accompanied by a re-hybridization of the atomic states. At the $\mathrm{P}$ atoms in the upper dimer a de-hybridization into $\mathrm{s}$ and $\mathrm{p}$ orbitals takes place, two of the latter contribute to back bonds. The $\mathrm{P}$ atoms of the lower dimer tend to a planar configuration with three $\mathrm{sp}^{2}$ hybrids and one $\mathrm{p}_{z}$ dangling orbital. Because the orbital energies of $\mathrm{Ga}$ are higher than those of $\mathrm{P}$, the majority of the P dimer states are occupied with two electrons. This holds for all states belonging to the upper dimer and for the $\sigma-, \pi$ - and $\pi^{*}$-dimer states of the lower pair (or, more strictly, mixed states of them and back-bond states). The $\mathrm{GaP}(001) \mathrm{p}(2 \times 2) \mathrm{td} 2$ surface is thus semiconducting due to the inequivalence of the top $\mathrm{P}$ dimers.

\section{Summary}

We have presented a comprehensive study of various geometries and stoichiometries proposed for P-rich $\mathrm{GaP}(001)$ surfaces. The calculated surface phase diagram confirms for an intermediate range of preparation conditions between Ga-rich and P-rich the $\beta 2(2 \times 4)$ structure known from $\mathrm{GaAs}(001)$ surfaces. For extremely P-rich preparation conditions we predict the occurrence of two phosphorous adlayers on top of a Gaterminated substrate forming a $\mathrm{p}(2 \times 2)$ translational symmetry. The $\mathrm{P}$ atoms in the uppermost atomic layer form inequivalent dimers. The accompanying threefold coordination of atoms in the second layer is reminescent of solid black phosphorus. For the geometries studied here we observe strong similarities between $\mathrm{GaP}$ and $\operatorname{InP}(001)$ surfaces.

Acknowledgements We are grateful for helpful discussion with Patrick Vogt and Norbert Esser. This work was supported by the Deutsche Forschungsgemeinschaft (Be 1346/10-1) and by grants of computer time from the John von Neumann-Institut Jülich. 


\section{References}

[1] Q.-K. Xue, T. Hashizume, and T. Sakurai, Prog. Surf. Sci. 56, 1 (1997).

[2] M. M. Sung and J. W. Rabelais, Surf. Sci. 365, 136 (1996).

[3] N. Oishi, F. ShoJI, A. Konishi, M. NAitoH, and S. NishigaKi, Surf. Rev. Lett. 5, 223 (1998).

[4] M. Naitoh, A. Konishi, H. Inenaga, S. Nishigaki, N. Oishi, and F. ShojI, Surf. Sci. 402-404, 623 (1998).

[5] N. Sanada, S. Mochizuki, S. Ichikawa, N. Utsumi, M. Shimomura, G. Kaneda, A. Takeuchi, Y. Suzuki, Y. Fukuda, S. Tanaka, and M. Kamata, Surf. Sci. 419, 120 (1999).

[6] M. Yoshikawa, A. Nakamura, T. Nomura, and K. Ishikawa, Jpn. J. Appl. Phys. 35, 1205 (1996).

[7] M. Zorn, B. Junno, T. Trepk, S. Bose, L. Samuelson, J.-T. Zettler, and W. Richter, Phys. Rev. B 60, 11597 (1999).

[8] A. M. Frisch, W. G. Schmidt, J. Bernholc, M. Pristovsek, N. Esser, and W. Richter, Phys. Rev. B 60, 2488 (1999).

[9] N. Esser, W. G. Schmidt, J. Bernholc, A. M. Frisch, P. Vogt, M. Zorn, M. Pristovsek, W. Richter, F. Bechstedt, Th. Hannappel, and S. Visbeck, J. Vac. Sci. Technol. B 17, 1691 (1999).

[10] K. Lüdge, P. Vogt, O. Pulci, N. Esser, F. Bechstedt, and W. Richter, Phys. Rev. B 62, 11046 (2000).

[11] L. Li, B.-K. Han, Q. Fu, and R. F. Hicks, Phys. Rev. Lett. 82, 1879 (1999).

[12] P. Vogt, Th. Hannappel, S. Visbeck, K. Knorr, N. Esser, and W. Richter, Phys. Rev. B 60, R5117 (1999).

[13] D. R. Hamann, Phys. Rev. B 40, 2980 (1989).

[14] K. ShIRAIShI, J. Phys. Soc. Jpn. 59, 3455 (1990).

[15] R. Stumpf and M. Scheffler, Comput. Phys. Commun. 79, 447 (1994).

M. Bockstedte, A. Kley, J. Neugebauer, and M. Scheffler, Comput. Phys. Commun. 107, 187 (1997).

[16] W. G. Schmidt, J. Bernholc, and F. Bechstedt, Appl. Surf. Sci., 166, 179 (2000).

[17] G.-X. Qian, R. M. Martin, and D. J. Chadi, Phys. Rev. Lett. 60, 162 (1988); Phys. Rev. B 38, 7649 (1988).

[18] D. R. LIDE (Ed.), CRC Handbook of Chemistry and Physics, 77th ed., CRC Press, Boca Raton (Florida) 1996.

[19] O. Pulci, K. Lüdge, W. G. Schmidt, and F. Bechstedt, Surf. Sci. 464, 272 (2000). 